\title{
An Examination of Pedestrian Trip Behaviour in Underground Pedestrian Systems
}

\begin{abstract}
Underground Pedestrian Systems (UPS) that provide alternative walking options for pedestrians have been implemented in a considerable number of cities across the world. These systems were found to have markedly improved the convenience and ease of city life in terms of transport, economic activities and social activities because they appear to be used throughout the day by a large number of pedestrians with different purposes. China has seen continuous UPS development over the past 30 years. However, as a relatively new form of pedestrian systems, usage patterns of UPS remain largely unexplored. In this research, surveys of pedestrians' trip behaviour related with UPS usage in Shanghai were conducted. Qualitative and quantitative data were collected and analysed. The research indicates that there are a diversity of users of UPS in terms of gender, age, education level, employment status and occupation, and personal income. Shopping and employment were two main reasons why respondents used the UPS. Subways and commerce are two vital factors related to UPS usage. These factors provide a functional basis for the UPS, and the UPS in turn creates both a catalytic and synergistic relationship that reinforces the performance of these factors.
\end{abstract}

\section{KEY WORDS}

Pedestrian; Trip behaviour; Underground Pedestrian System; Shanghai

\section{Introduction}

Underground pedestrian systems (UPS), a phenomenon of comprehensive utilisation of urban underground space (UUS) in central areas of mega cities, have been developed around the world during the past century. Ancient underground systems that developed before the $20^{\text {th }}$ century were constructed mainly for the purposes of concealed shelter and defence in times of civil disturbance or war. It was not until the $20^{\text {th }}$ century that modern multi-functional UPS, typically integrating mass transit with retailing concourses and commercial land uses, became a commonplace urban development feature in many of the world's major metropolises.

UPS development has occurred for various reasons. Influencing factors in both natural and built environments have contributed to its inclusion in major cities worldwide (Cui et al., 2013a). In cities with severe weather conditions such as Toronto (Canada), a cold winter city and Oklahoma City (United States), a windy city, UPS have provided a climate-controlled environment for year round social, entertainment, retail and commercial activities. In dense urban settings such as Tokyo (Japan) and Shanghai (China), UPS have provided opportunities for much more comprehensive and intensive usage of urban spaces, by accommodating multiple urban functions comparable to those which occur at street level.

Since China's economic reforms launched in 1978, and particularly over the past decade, the capacity of UUS utilisation in China has increased rapidly. The scale and growth rate of UUS development in major cities in China, such as Shanghai and Beijing, have drawn global attention. In Shanghai, nearly 40 million square metres $\left(\mathrm{m}^{2}\right)$ of UUS were developed up to early 2010. In Beijing, as of late 2006, there were more than 30 million $\mathrm{m}^{2}$ of UUS, and it was estimated that by 2020 , UUS capacity will increase to 90 million $\mathrm{m}^{2}$, or $5 \mathrm{~m}^{2}$ per person (He et al., 2012).

In China's megacities, UPS were developed in built-up districts to counteract problems with land use, urban transport and the environment and to face the challenge of building the image of a metropolis and improving the quality of urban life. This has been evident in many downtowns, such as those in Beijing, Shanghai and Guangzhou. In protected historic areas, the use of underground space can alleviate tension between urban development and historic protection by providing effective measures for the protection of valuable sites. For example, in Bell Tower in Xi'an, the utilisation of UPS protected historical architecture and redeveloped the city centre (Zhang et al., 2009). In addition, underground public spaces were built in newly urbanising districts as a symbol of modernity. Cases of UUS in the newly urbanising districts can be found in Beijing's Zhongguancun and Yizhuang; Guangzhou's Zhujiang New Town; Shanghai's Jiading and Baoshan Districts; Hangzhou's Qianjiang CBD; Shenzhen's Bao'an District; Zhengzhou's Zhengdong New Area; Wuhan's Wangjiadun CBD; and Tianjin's Binhai New Area (He et al., 2012; Qian, 2005). 
Shanghai is a representative city of underground space development in China. It is a pioneer city of UUS development in China. Shanghai has been experiencing dramatically rapid urbanisation and, accordingly, the severe urban problems emerging from the process have driven its underground space utilisation. Moreover, Shanghai has the longest subway system of world cities, thereby providing a catalyst for UUS/UPS development. In addition, as the core of the fastest growing economy of the Yangtze River Delta, Shanghai has the economic capability to explore underground use for urban development.

\section{Trip Behaviour of UPS Users}

UPS, forming a second level and weather protected walking environment, has the potential to affect people's travel behaviour (Cui et al., 2013b). Previous research has indicated that there have been three main purposes of space utilisation of UPS, namely public transport, shopping and entertainment activities (Zacharias and Xu, 2007). The essential function of UPS is to facilitate accessibility by walking (the transport function) whilst a secondary function is to provide integration with commercial functions, retail functions and convenient mass public transport (typically the rail subway). There has been little research focusing on pedestrians' trip behaviour related with the use of UPS in the world, except for North American cities with intensive UPS development, such as Toronto and Montreal in Canada and Chicago in the United States (US). The reason for this past research interest is the desire to understand the usage patterns of UPS, given that UPS are consciously designed pedestrian environments that typically integrate indoor underground spaces, with public transit and economic functions in a centralised or city centre urban setting.

To explore usage patterns of UPS, previous research has been concerned with three main questions: (1) who are the UPS users; (2) how people access UPS; and (3) where the pedestrian flows generate. Robertson (1988) conducted an investigation and comparison of the usage patterns of skywalk systems ${ }^{1}$ of five midwestern US cities: Cincinnati, Des Moines, Duluth, Minneapolis and Saint Paul. Robertson's research identified that it was generally the social elite that were the dominant users. The respondents of his survey perceived that the typical skywalk user was a female white-collar worker earning a high income of over 50,000 US Dollars a year (1988 values). However, this study focused on skywalk systems, while evidence from UPS has not been recorded.

Some research has focused on how people access UPS. Byers' (1998) survey of grade separation pedestrian systems (including UPS, skywalk system or a combination of the both) in Toronto, Minneapolis and Houston examined the land use of the area where UPS and skywalk systems are located. He assumed that since various efforts had been committed to connect commercial and retail activities to parking facilities and public transit through the construction of UPS and skywalk systems, these strongly influenced the general public's moves around the city centre. UPS were found to have provided convenient public transport and accessible shopping environments, which persuaded employees to spend more of their leisure time in the city. However, the research is mainly from qualitative analysis while quantitative evidence needs to be updated.

Pedestrian flows within UPS have also received some attention. Zacharias (2000) used surveys and experiments to explore the behaviour and distribution of pedestrian flows within an UPS to help provide an understanding of how and why these pedestrian flows occur. His research identified that the distribution of pedestrians in UPS is generally stable. Planned public and retailing activities appeared to have had only a moderate effect on pedestrian distributions in the UPS (Zacharias, 2000). New large facilities were found to have had insignificant influences on pedestrians and their movement patterns (Zacharias and Schinazi, 2003). It would be useful to include more facilities such as shopping malls and streets, department stores and other buildings to see their impacts on attracting pedestrian flows.

What is more important, while UPS experiences from North American cities have been well documented, the UPS experience elsewhere, such as in East Asian countries, has not been a focus of research. It is therefore of significant importance to fill the gap on the usage patterns of UPS in East Asian countries, to identify what lessons could be learned and what could be useful elsewhere. With strong support from central and local governments, many Chinese cities have applied UPS strategies, with Shanghai being viewed as a pioneer of UPS utilisation - its UPS were introduced from the early 1990s. This has provided a good opportunity to conduct detailed research of usage patterns of UPS in Shanghai. The findings from this research would be

\footnotetext{
${ }^{1}$ In the research of North America where UPS and skywalk systems received extensive development, the underground and elevated pedestrian systems were sometimes investigated together as grade separation pedestrian systems. But literature specifically targeting the development and usage patterns of UPS is scarce.
} 
useful to planning authorities when they develop UPS and when considering how to plan, design and operate UPS for better service.

\section{Methodology}

This research selected a case study in Shanghai, China, to effectively investigate UPS. Exemplars of UPS in Shanghai in terms of an optimum location (for accessibility to major pedestrian trip generators), development, duration and scale include: UPS in People's Square, Jing'an Temple and Xujiahui. The locations and comparative structures and sizes of the three UPS are shown in Figure 1. The three UPS have various development initiatives. Since the 1950s, Shanghai's People's Square UPS benefited from its official declaration by the national government as having core status as the People's Square area. People's Square is a typical example of city centre redevelopment mainly relying on subway development. Somewhat similar to People's Square, the Jing'an Temple UPS development is based around an underground railway station. Xujiahui UPS, likewise, is based around an underground railway station. However, commercial buildings above the UPS and adjacent to it are another dynamic component of UPS development.

A review of the research methodologies used in similar research projects utilising empirically derived research was conducted, such as Barker (1986), Byers (1998), Robertson (1987), and Belanger (2007), and a mixed method of quantitative and qualitative survey techniques was determined for application. The benefits of using integrated quantitative and qualitative research methods have the potential to increase knowledge on the research topic because the respective characteristics of the two methods, when combined, can compensate for each other's merits and shortcomings (Valadez and Bamberger, 1994). For this research, the analysis could be addressed on different levels by employing an integrated approach: first, an appropriate sampling methodology is likely to yield research findings that are representative of the population being studied; second, the sample selections were conducted with reference to earlier survey research to enable direct comparisons and contrasts to be made between previous work and the current survey's findings; and third, statistical analysis could be applied to generalise the characteristics of respondents with their respective social conditions, complemented by qualitative research techniques that provide explanations in much greater depth than what could ordinarily be achieved with a basic statistical analysis of respondents (Bamberger, 2000).

The methodology of this research included three data collection methods. The initial method was textual analysis of publications, websites and reports regarding Shanghai's economy and transport for the purpose of enhancing an understanding of the Shanghai context of UPS development. The second method was an observational study of the three UPS in Shanghai to provide an understanding of pedestrian activity and features of the system such as pedestrian usage, design, activities and unique characteristics. Third, a questionnaire survey of pedestrians in three UPS in Shanghai was conducted. It concerned the trip behaviour of the respondents accessing and leaving UPS that was gleaned from information regarding a respondent's trip characteristics (i.e. trip purposes, travelling modes and duration and trip destinations). Before the questionnaire survey was implemented, a pilot survey was undertaken to test the effectiveness of the questionnaire. The questionnaire was refined and finalised based on the feedback from 20 respondents. Questionnaires were then conducted face to face in the three locations in January and February of 2011 by the main researcher: 66 in People' Square (31.8\%), 71 in Jing'an Temple (34.1\%) and 71 in Xujiahui Area (34.1\%). The questionnaire applied Almond's method (named after the British traffic engineer who devised it) as the sampling strategy to achieve a randomised sample, with targeting via invitation to participate in the survey of every second pedestrian passing by, after the interviewer had completed a questionnaire. The survey yielded 208 complete questionnaires: 145 respondents (69.7\%) on weekdays and 63 (30.3\%) on weekends. The quantitative data were then systematically coded and arranged using Microsoft Excel and Statistical Package for Social Sciences (SPSS). The analysis of the quantitative data included two stages: (1) a descriptive analysis of the statistical data; and (2) the SPSS analysis of correlations (such as the correlation of the trip duration with demographic variables). The qualitative data from textual analysis and observation then were analysed to provide an additional viewpoint of the findings from the quantitative analysis.

Insert 'Figure 1' here.

\section{Description of Shanghai's context}

As the commercial and financial centre of China, the economy of Shanghai continues to grow. Since the launching of the economic reforms in 1978, the growth rate of Gross Domestic Product (GDP) in Shanghai 
has remained at a sustained high level, especially during the past decade. The average annual growth of GDP was about $10.5 \%$ over the past three decades, averaging $11.6 \%$ annually during the past decade (Shanghai Municipal Statistics Bureau, 2011). Exceptionally strong economic performance since the economic reforms based on trade, finance, manufacturing and services, has enabled Shanghai to undertake massive investment in urban development and has created favourable conditions for the local government and developers to opt for underground space development and underground transit networks.

As Shanghai experiences rapid motorisation, the number of private automobiles has increased rapidly over the past two decades. Automobile ownership in Shanghai had already reached around 1.7 million by 2010, a two-fold increase within a decade. The increase in the number of automobiles in Shanghai is remarkable, especially in private cars, with nearly an eleven-fold increase within a decade (Shanghai Municipal Statistics Bureau, 2011).

$\mathrm{Li}$ and $\mathrm{He}$ (2008) studied the changing structure of transport modal shares in Shanghai from 1986 to 2004, with a trend away from walking ( $41 \%$ down to $29 \%$ ) to automobiles (increasing from less than $1 \%$ to $16.5 \%$ ). According to Figure 2, although walking and cycling are still the dominant transport modes in Shanghai city and city proper, car transport had accounted for around 20\% of trips in Shanghai in 2008. The rapid increase in automobile transport has challenged the priority traditionally given to walking. The urban realm for these transportation modes has been eroded with side effects including pollution, car accidents and congestion. Additionally, the inadequacy of pedestrian facilities and inefficient traffic management has disadvantaged pedestrians by failing to prevent conflicts between automobiles and pedestrians ( $\mathrm{Li} \& \mathrm{He}, 2008$ ).

Insert 'Figure 2' here.

Although car transport has resulted in a remarkable increase in Shanghai's visible surface level transport infrastructure, subway transport has played an increasingly important role in public transport with the increase in the number and length of operational lines. In 2008, subway transport had an $18 \%$ share of public transport in Shanghai city (Shanghai Municipal Committee of Rural and Suburban Construction and Transportation, 2009). In 2010, the volume of passenger traffic of the subway reached 1.9 billion person-trips, 6.7 times that the volume in 2001 (see Figure 3).

Insert 'Figure 3' here.

The data of Shanghai's transport modal split indicates that first, walking transport has seen an inevitable decline with the phenomenal expansion of automobile transport; and second, public transport (subway with its gradual improvement as representative) may be more important in the long term to Shanghai achieving an efficient and effective transport system. Shanghai is gradually implementing a public transport priority strategy with a strong focus on subway transport. According to Shanghai Metro Planning, the subway system in Shanghai will ultimately form a network length of 480 kilometres serving 200 underground stations concentrated within Shanghai's central area. Modal paths of 'walking + public transport + walking' and 'public transport + walking + public transport' appear destined to become vital components of urban transport (Li \& He, 2008). Thirdly, the development of the subway system and the catalytic effects of the subway's construction and UUS utilisation provide valuable opportunities for UPS development. The incomplete pedestrian facilities and services at street level provide an opportunity for UPS to allow the creation of complete pedestrian systems, thereby increasing the accessibility and comfort of the pedestrian experience.

\section{Usage patterns of UPS}

The three UPS in Shanghai were investigated through a field survey. The data from the field survey were analysed and discussed, examining the usage patterns of UPS users specifically according to social-economic profile of the users of UPS and travel behaviour related with the usage of UPS.

\subsection{Demographic profiles of the users of UPS}

A detailed sample profile of the 208 respondents is shown in Table 1. Female respondents accounted for $55.8 \%$ of the total respondents while male respondents only accounted for $44.2 \%$ of the total survey cohort. The shopping streets and malls connected by the UPS appear to be more attractive to women. With respect to age, about $84 \%$ of respondents were aged between 18 and 34 years. With older age cohorts, the number of users appeared to reduce significantly. Respondents of more than 65 years old only accounted for $0.5 \%$ of the total survey cohort. This could be because escalators and lifts were not available near some of the main entrances and some lifts were hard to locate, either underground or at street level. Facilities for the elderly and 
disabled possibly need an improvement within the Shanghai UPS to facilitate the usage by seniors and the disabled.

Insert 'Table 1' here.

In North American research, the typical skywalk user was perceived to be a female white-collar worker who has a high income (Robertson, 1988). It is interesting to examine whether this phenomenon existed in Shanghai's UPS. Figure 4 gives an illustrative breakdown of the respondents by age and gender and provides some basic characteristics of the respondents. The number of female respondents is more than male respondents, and this phenomenon is more obvious within the 18-24 and 25-34 age groups.

Insert 'Figure 4' here.

In relation to education level, UPS users that hold undergraduate degrees accounted for $42.3 \%$ of those surveyed, the first largest survey cohort, while those who graduated from junior colleges comprised the second largest survey cohort, accounting for $28.8 \%$ of those surveyed (see Table 1). With regard to employment status and occupation, respondents appeared to come from a variety of social backgrounds. The UPS users were a combination of full time employees, part time employees, retired people, students, home workers and unemployed people. The great majority of respondents (80\%) were full time employees. One possible reason is that many people take public transport that is integrated with the UPS, to access their company offices located in the downtown or near the UPS. Only 3.4\% of respondents were unemployed, which is slightly lower (0.9\%) than Shanghai's registered unemployment level in 2009 (Shanghai Municipal Statistics Bureau, 2010). Students, part time employees, retired people and home workers accounted for $6.2 \%$ to $1.4 \%$ of total respondents. About $36 \%$ of respondents' work belonged to the commercial and service sectors. This could be associated with the commercial spaces and entertainment facilities that are connected with the UPS. Professional job holders accounted for $18.3 \%$ of respondents while manager/administrator, clerk and other types of occupation accounted for $13 \%$ to $6.2 \%$ of total respondents.

When examined according to income (see Table 2), UPS users that have monthly income of between 2,000 and 3,000 Yuan (22.6\%) and those that have monthly income of between 3,000 and 4,000 Yuan (24.0\%) are the two largest survey cohorts. The average monthly income of Shanghai's residents was 2,400 Yuan in 2009 (Shanghai Municipal Statistics Bureau, 2010) while in this survey, respondents whose income was over 3,000 Yuan per month accounted for $58.8 \%$ of the total survey cohort. The UPS users that participated in the survey appear to have comparatively high income levels. The phenomenon of high income UPS users possibly is associated with a predominantly youthful age cohort of users in the Shanghai UPS survey, members of which who generally have a high level of education attainment.

Figure 5 gives an illustrative breakdown of the respondents by personal income and gender. The number of female respondents is more than male respondents, especially in the groups with personal income per month between 2,000-3,000 Yuan and 3,000-4,000 Yuan. However, it is hard to conclude whether or not female respondents have a higher income level than that of male respondents because, with the increase in income level, the number of male respondents tended to be greater than female respondents.

Insert 'Figure 5' here.

\subsection{Trip purpose}

Amongst the 208 respondents, Figure 6 shows that the primary reasons for visiting the UPS were shopping (39.4\%) and employment (27.9\%). This finding is similar to those of other surveys of UPS user profiles: the main users of Shanghai Xiao Lujiazui UPS are those using it for entertainment purposes (including tourists and shoppers) and employees (Zhang \& Huang, 2008); among the users in Montreal's UPS, employees dominated followed by those who visited commercial facilities underground and at street level (Boisvert, 2004). Among the respondents of the Shanghai survey, with the exception of shoppers and employees, residents, tourists and 'other' accounted for $8 \%$ to $14 \%$ of respondents. UPS users exhibit mixed urban characteristics. This phenomenon has resulted from increasing urbanisation and is also an outcome of the efficiency of subway transportation in bringing people from across metropolitan Shanghai into the UPS (Zhang \& Huang, 2008).

Insert 'Figure 6' here.

North American research has found differences in the usage of UPS on weekdays and weekends in different cities. Byers's research (1998a) compared three North American cities and found that there was less activity on weekends than on weekdays in UPS and skywalk systems in city centres. On weekends, activities were concentrated in the peripheral districts of downtowns that were not occupied by UPS and skywalk systems. 
Kong and Furman (2006) argued that in Toronto, organised events and informal uses occurred there, such as using the concourse as a mini indoor football stadium or the empty food courts on weekends as study corners. In order to determine the difference between usage on weekdays and weekends, the respondents were classified according to the primary reason for coming to the UPS on weekdays/weekends. As shown in Figure 7 , on weekdays, shopping was the most important primary reason for visiting the UPS, accounting for $42.1 \%$ of responses. This is followed by employment (25.5\%) and tourism (13.8\%). On weekends, employment and shopping were two equally important primary reasons for coming to the UPS, with $33.3 \%$ of respondents for each reason. Tourists tend to be more likely to use an UPS on weekdays (13.8\%) than on weekends (3.2\%). The results suggest that differences exist in the use of an UPS in Shanghai on weekdays and weekends: first, shopping is a primary reason for visiting the UPS on both weekdays and weekends, although shopping activity is more important in the UPS on weekdays; second, employment is more important in the UPS on weekends than weekdays - this possibly relates to the decrease of shoppers on weekends and the extended working time on weekends of employees of commercial and entertainment facilities that are connected with the UPS; third, on weekends, residents are more likely to stroll in the UPS for leisure.

Insert 'Figure 7' here.

\subsection{Transportation modes and duration}

All of the respondents were asked for their destinations, duration of stay in the UPS, transport modes to access the UPS and trip duration accessing the UPS; while for those with a destination other than the UPS, they were asked their reasons for walking in the UPS, transport modes used for leaving the UPS and the duration of their next trip. The destination for $60.6 \%$ of respondents was the UPS, while the balance of respondents $(39.4 \%)$ used the UPS to transfer to other places (see Table 2). The home was the most significant origin of trips, accounting for $53.9 \%$ of responses. About $67.8 \%$ of respondents stayed in the UPS for less than 10 minutes, which may be related to the large rate of transfer (39.4\%) within the UPS. From the survey, it was also found that many respondents, when commencing the questionnaire, had just arrived in the UPS. Notwithstanding this, $5.8 \%$ of respondents stayed longer than two hours in the UPS, due to their work commitments.

Insert 'Table 2' here.

The overwhelming majority of respondents accessing the UPS relied on transit (95.2\%) including the subway, bus and walking, among which, $68.2 \%$ of respondents came to the UPS by subway, followed by walking with $15.4 \%$ and bus with an $11.5 \%$ share. Those who travelled by car, taxi, bicycle or other transport modes were very rare, accounting for less than $5 \%$ of respondents in aggregate. The results indicate that firstly, transit is the most significantly important transport mode to achieving underground walkway use. Accordingly, the walkway system should be responsive to the needs of the transit rider by providing the necessary linkages to transit facilities such as subway and bus. Second, the relationship between UPS pedestrians and subway transport highlights the importance of well-integrated public transport facilities; third, the results indicate that UPS pedestrians rarely choose car or taxi to travel. The reason for this phenomenon is possibly because the convenience and accessibility between public transport and the UPS makes use of those modes redundant. From this perspective, UPS could have a powerful effect in promoting usage of public transport.

With regard to the duration of the travel time from a respondent's origin to the UPS (see Table 2), overwhelmingly, $96.6 \%$ of respondents had a travel time of less than one hour; nearly $60 \%$ of travel time was within half an hour. About $19.7 \%$ of respondents began their trip nearby, with trip duration of less than 10 minutes. Only $3.4 \%$ of respondents stated that they spend more than one hour in travelling time to reach the UPS. The majority of the trips that the UPS attracted were short- or medium-duration trips.

For those with a destination other than the UPS (see Table 3), although seven options were provided to the respondents, $70.7 \%$ of respondents cited the main reason for them walking within the UPS was to access travel on the subway; a little less than one-tenth of respondents chose the UPS for its convenience. By contrast, the primary reasons for walking within North American UPS were: (1) no safe alternative aboveground routes to use; (2) a liking for indoor pedestrian environments; and (3) familiarity with the underground network (Corbett, 2009; Dillon, 1985; Robertson 1993a, b; Wang \& Liang, 2010). However, these reasons were seldom nominated by the respondents in Shanghai's UPS. This could be because those respondents were transit riders in the UPS; thus, their primary reasons for using the UPS differ from those who target activities specifically within the UPS.

Insert 'Table 3' here. 
According to Table 3, a little less than one-half of respondents who leave the UPS did so as a pedestrian, followed by those who left by subways $(31.7 \%)$, while the remaining $19.5 \%$ left by bus or taxi. Nearly half of the respondents arrived at their destinations within 10 minutes and $84.1 \%$ within half an hour. This suggests that it is important to maintain the completeness of pedestrian networks in the immediate environment of UPS, such as those within ten minutes walking distance.

\subsection{Correlations between trip duration and demographic variables}

To provide further insight into trip durations related to the use of the UPS, Spearman's correlation test was undertaken to examine the associations of some demographic variables with trip durations when accessing the UPS. As shown in Table 4, there is a statistically significant negative correlation between the trip duration accessing the UPS (-.172) and personal income, within a 95\% confidence interval. Respondents who have a higher personal income had shorter trip duration than did those respondents who have a lower personal income. The reason for this phenomenon is possibly that those who have a higher personal income tend to have travel alternatives that minimise the trip duration (such as motorised transport instead of non-motorised transport modes).

Insert 'Table 4' here.

Figure 8 presents the influence of personal income on trip durations accessing the UPS. Among all mean scores, the variable 'trip duration accessing UPS' received the highest score (2.368) in the '1,000-2,000 Yuan' group. As mentioned, according to the Shanghai Municipal Statistics Bureau (2010), the average monthly income of residents was 2,400 Yuan in 2009. One possible explanation is that people with low personal incomes are less likely to afford more expensive transport options, which results in having longer travel duration to access the UPS.

Insert 'Figure 8' here.

\subsection{Generators of pedestrian flows in UPS}

To understand where pedestrian flows are generated in UPS, an investigation of destinations of trips related with UPS usage was undertaken. As indicated, the UPS users in Shanghai survey can be divided into two groups: those whose destination was an UPS (60.6\%) and those who transferred at an UPS (39.4\%) either by transiting through an UPS or by transfer via a subway. Table 5 shows the destinations of respondents who indicated their final destination was within the UPS. Almost half of the respondents (45.2\%) had a destination of department stores/shopping malls connected with the UPS, followed by underground shopping streets/retailing shops (13.5\%) and underground shopping malls (11.1\%). This corresponded with the composition of respondents in the UPS, amongst which shoppers (39.4\%) accounted for the majority. The UPS relied on its commercial function to a large extent to ensure its survival. The results confirmed Bhalla and Pant's (1985) findings of pedestrian trips within the skywalk system in the CBD of Cincinnati that the floor space area for retail use was one of the four most significant variables.

Insert 'Table 5' here.

According to the comparison between the three UPS (see Table 5), the land use of each UPS were the main characteristics attracting the majority of respondents: the department stores and shopping malls in Xujiahui UPS; the underground shopping streets in the People's Square UPS ${ }^{2}$; and a combination of a department store and a historical heritage attraction - Jing'an Temple - in Jing'an UPS. It is worth noting that the temporary stall $^{3}$ is a phenomenon in Shanghai's UPS that is distributed in underground walkways and entrances/exits in the three UPS. In Jing'an Temple UPS, $6.8 \%$ of the respondents nominated temporary stalls as their destination, highlighting the strong influence of temporary stalls in Jing' an Temple UPS. In addition, 8.8\% of respondents in People's Square UPS had as their destination People's Square at street level, which contrasted

\footnotetext{
${ }^{2}$ The number of respondents who visited the underground shopping streets in People's Square UPS is the highest among the three locations because the People's Square UPS includes three underground shopping streets with different and unique features.

${ }^{3}$ A temporary stall is a phenomenon within Shanghai's UPS. The prices of merchandise in the temporary stalls are very competitive. The types of merchandise that temporary stalls provide are cheap, diverse and convenient. The stall owners are also very approachable and satisfy consumer demand from Shanghai's floating population, residents/employees with low incomes and students. But temporary stalls could challenge the desire to maintain order, a uniformity of visual appearance and the viability of formal commercial components within the UPS.
} 
with respondents in Jing' an Temple UPS, none of whom went to the Jing' an underground concourse, a similar plaza in terms of function and its design intent for public usage.

\section{Conclusions}

Shanghai, a mega city of 23 million people, is situated at the heart of the Yangtze River Delta, and has some of the most rapid economic growth in China. Shanghai has a cosmopolitan character, and it is at the forefront of developing a functionally complex, sophisticated and technologically advanced metropolitan image of modern China in the 21st century. The development of Shanghai's UPS arose out of common place challenges that many modern cities around the world have to contend with, such as a shortage of land, large scale city centre redevelopment and major transport infrastructure improvements. In addition however, Shanghai has also experienced a rapidly changing social dynamic with rapid economic growth and urbanisation, urban sprawl, hyperbolic growth rates of car ownership and subway construction on a large scale.

With a favourable urban context, especially the construction of subway systems that largely promote the formation of UPS, UPS in Shanghai has been developed to a large extent. This has provided a good opportunity to conduct detailed research of usage patterns of UPS in Shanghai. The demographic profiles of respondents of Shanghai's UPS (N=208) represented a diversity of users in terms of gender, age, education level, employment status and occupation, and personal income, but UPS users are more likely to be female, young, highly educated, in full time employment and with high incomes. According to the study, females (accounting for 55.8\% of respondents) are more likely to use UPS than males $(44.2 \%)$ due to the attractiveness (to women) of shopping streets and malls connected to the UPS; young users aged between 18 and 34 years ( $84.1 \%$ of respondents) appear to be more likely to use UPS than older age cohorts, with the number of users declining significantly with age; users tended to rarely use UPS. About $75.9 \%$ of UPS users had high levels of education (a junior college level of education or higher). Nearly $80 \%$ of respondents were full time employees while the rest were the combination of part-time employees, retired people, students, home workers and unemployed people. A large number of respondents' (36.0\%) work related to the commercial and service sectors; UPS users that participated in the survey appeared to have comparatively higher average monthly income levels than the residents of Shanghai.

Shopping and employment were two main reasons why respondents visited the UPS $(\mathrm{N}=208)$. Respondents' primary reasons for visiting the UPS in Shanghai were either shopping (39.4\%) or employment $(27.9 \%)$, which is a similar result to other surveys of UPS user profiles in Shanghai and Montreal (Boisvert, 2004; Zhang \& Huang, 2008); differences exist in the use of an UPS in Shanghai on weekdays and weekends - shopping activity is more important in the UPS on weekdays, while employment is more important in the UPS on weekends. This is possibly related to the extended working time on weekends of employees of commercial and entertainment facilities that are connected with the UPS; the majority of the respondents (75.5\%) came to the UPS without something specifically attracting them but largely for functional reasons.

Subways and commerce are two vital factors related to UPS usage $(\mathrm{N}=208)$. These factors provide a functional basis for the UPS, and the UPS in turn creates both a catalytic and synergistic relationship that reinforces the performance of these factors. For $95.2 \%$ of respondents, transit was the most significant transport mode in achieving underground walkway use; this number included $68.2 \%$ of respondents who came to the UPS by subway. The convenience and accessibility between public transport and the UPS makes use of those modes preferable. This is very different from North American cities such as Winnipeg where private car transport was the dominant mode for accessing UPS and the skywalk system (Eady, 1990). For those who transferred within the UPS in the survey $(\mathrm{N}=82), 70.7 \%$ of respondents cited that the main reason for them walking within the UPS was to access travel on the subway. The most important destinations for respondents who indicated that their final destinations were within the UPS $(\mathrm{N}=126)$ were department stores/shopping malls connected with the UPS (45.2\%), underground shopping streets/retail shops (13.5\%), and underground shopping malls $(11.1 \%)$. The featured commerce patterns in the three UPS (such as three underground shopping streets in People's Square UPS, department stores and an underground shopping mall in Jing'an Temple UPS, and massive department stores and malls in Xujiahui UPS) attracted a majority of the survey participants whose destinations were within the UPS.

In addition to the characteristics that reflected in the examination of usage patterns of Shanghai's UPS, the research also uncovered problems in relation to people's use of the system. For example, escalators and lifts were not available near some of the main entrances and exits, and some lifts were hard to locate either underground or at street level. This could be inconvenient for those with children, the elderly and disabled 
people using the UPS. This appears to be one reason why the majority of UPS users belonged to a younger age cohort ( $84.1 \%$ of respondents were $18-34$ years old). Moreover, currently, the subway system is the only public transport system that completely integrates with the UPS in Shanghai; for public bus services, pedestrians normally need to transfer to a bus at street level without the benefit of doing this through a transport interchange facility (Jing'an Temple UPS has recently developed a $255 \mathrm{~m}$ long underground walkway connecting Public Transportation Interchange). Comparing large UPS globally, the scale and functionality of Shanghai's UPS is still developing.

UPS are a relatively new topic in urban and transport studies, and therefore there is clearly a need for more research on this topic. What has emerged from the findings of this paper provides directions for future research in this field. For example, there has been little evidence-based research of the usage patterns and impacts of UPS in cities other than North American cities. Previous research included extensive debates on a variety of issues in terms of the functionality of the UPS and its urban impacts. With future urban growth framed within an agenda of sustainable urban development, there is a strong need for new knowledge that further examines the functionality and impacts of UPS. In the case of Shanghai, this research can be viewed as an initial exploration using evidence-based research to examine the functionality and impacts of UPS. Moreover, there is a need to study the impacts of UPS over different time periods. With changes in the city's urban development, the impacts of an UPS on people's trip behaviour would change over time. Accordingly, systematic data collection and analysis post-completion of the UPS as well as a comparison of the impacts of UPS in different periods would be essential to enhancing an understanding about the evolution of UPS impacts over time and as the city evolves. Considering that UPS are infrastructure of considerable longevity (Montreal's UPS has been operating for over 50 years), future research will be required that includes analysis of long-term data and impacts in Shanghai and other cities.

\section{Acknowledgements}

We would like to thank the anonymous reviewers and the editor for their valuable comments and suggestions.

\section{References}

Bamberger, M., 2000. Integrating quantitative and qualitative research in development projects. The International Bank for Reconstruction and Development/The World Bank, Washington, D.C.

Barker, M.B., 1986. Toronto's underground pedestrian system. Tunnelling and Underground Space Technology 1 (2), 145-151.

Belanger, P., 2007. Underground landscape: The urbanism and infrastructure of Toronto's downtown pedestrian network. Tunnelling and Underground Space Technology 22 (3), 272-292.

Bhalla, M.K., Pant, P.D., 1985. Pedestrian traffic on Cincinnati skywalk system. Journal of Transportation Engineering 111(2), 95-104.

Boisvert, M., 2004. Underground city of Montreal and its influence on the ground commercial business. Underground Space 24(4), 551-553 (in Chinese).

Byers, J., 1998. The privatization of downtown public space: The emerging grade-separated city in North America. Journal of Planning Education and Research 17(3), 189-205.

Corbett, M.J., 2009. Evolution of the second-story city: the Minneapolis skyway system. Environment and Planning B: Planning and Design 36 (4), 711-724.

Cui, J., Allan, A., Taylor, M., Lin, D., 2013a. Underground pedestrian systems development in cities: influencing factors and implications. Tunnelling and Underground Space Technology 35, 152-160.

Cui, J., Allan, A., Lin, D., 2013b. The development of grade separation pedestrian systems: a review. Tunnelling and Underground Space Technology 38, 151-160.

Dillon, D., 1985. Dallas: a case study in skyway economics. Design Quarterly 129, 25-28.

Eady, R.M., 1990. The downtown pedestrian environment: an examination of Winnipeg's weather-protected walkway system from a user's perspective. Master of City Planning thesis, Department of City Planning, University of Manitoba.

He, L., Song, Y., Dai, S., Durbak, K., 2012. Quantitative research on the capacity of urban underground space - the case of Shanghai, China. Tunnelling and Underground Space Technology 32(5), 168-179.

Kong, T., Furman, A., 2006. Tunnel lives, viewed 3 Oct 2012, <http://www.surf.salford.ac.uk/cms/resources/uploads/File/Going\%20Underground/Thomas_Kong.pdf>.

Li, Q., He, L., 2008. Planning for pedestrian transport in central city of Shanghai (in Chinese: 'shang hai shi zhong xin cheng bu xing jiao tong gui hua yan jiu’). Shanghai Construction Science and Technology (1), 22-25. 
Qian, Q., 2005. Urban underground space utilization and present condition in China (in Chinese: 'zhong guo cheng shi di xia kong jian kai fa li yong xian zhuang, zhan wang he si kao'), opening speech of the 4th International Symposium on Underground Freight Transport (ISUFT2005), Shanghai, Oct 20-21.

Robertson, K.A., 1987. Pedestrian skywalks in Calgary, Canada: A comparison with US downtown systems. Cities 4 (3), 207-214.

Robertson, K.A., 1988. Pedestrian skywalk systems: Downtown's great hope or pathways to ruin? Transportation Quarterly 42 (3), 457-484.

Robertson, K.A., 1993a. Pedestrians and the American downtown. The Town Planning Review 64(3), 273-286.

Robertson, K.A., 1993b. Pedestrianization strategies for downtown planners - skywalks versus pedestrian malls. Journal of the American Planning Association 59(3), 361-370.

Shanghai Municipal Committee of Rural and Suburban Construction and Transportation 2009. Shanghai annual transport report 2008 (in Chinese: shang hai shi zong he jiao tong nian du bao gao 2008), Shanghai, viewed 7 Jan 2013, <http://www.scctpi.gov.cn/reports.asp?info_parentid=29\&info_id=1764>.

Shanghai Municipal Government 2011, Actual routes of metro lines within urban area, Shanghai, viewed 12 May 2012 , <http://live.shanghaidaily.com/images/guide/shanghai-map-b.gif>.

Shanghai Municipal Statistics Bureau 2010. Shanghai statistical yearbook 2010, China Statistics Press, Beijing.

Shanghai Municipal Statistics Bureau 2011. Shanghai statistical yearbook 2011, China Statistics Press, Beijing.

Valadez, J.J., Bamberger, M., 1994. Monitoring and evaluating social programs in developing countries: A handbook for Policymakers, Managers, and Researchers. The World Bank, Washington, D.C.

Wang, Y., Liang, L., 2010. Subterranean city: The introduction of Pedway in Chicago. Urban Planning International 25 (1), 95-99 (in Chinese).

Zacharias, J., 2000. Modeling pedestrian dynamics in Montreal's underground city. Journal of Transportation Engineering 126 (5), 405-412.

Zacharias, J., Schinazi, V., 2003. The impact of an entertainment retrofit on the performance of a shopping center. Journal of Shopping Center Research 10 (1), 29-44.

Zacharias, J., Xu, M., 2007. The underground system as economic generator for Montreal's central city. Urban Planning International 22 (6), 28-34 (in Chinese).

Zhang, J., Huang, J., 2008. Investigation and research on the underground walking system of small Lujiazui area in Shanghai. Environmental Architecture 4, 164-166 (in Chinese).

Zhang, P., Chen, Z., Yang, H., Wang, H., 2009. On utilization of underground space to protect historical relics model. Tunnelling and Underground Space Technology 24(3), 245-249. 